\title{
SOLUTION OF THE INVERSE PROBLEM FOR CYLINDRICAL INCLUSION FRAGMENT FORM DEFINITION
}

\author{
G.R. Shamsutdinova, S.V. Viktorov \\ Sterlitamak Branch of Bashkir State University, Sterlitamak, Russia
}

\begin{abstract}
We focus on the direct and inverse problems in this work. The solution of the inverse problems for deformation of extended cylindrical bodies comes to finding out A. N. Tikhonov's functional regularization extremal. It is based on the solution of the direct problem for point source field in a homogeneous half-space with a cylindrical inclusion by the method of integral representations.
\end{abstract}

Keywords: direct and inverse problems of electric exploration, method of integral representations, configuration method, Green's function, regional task.

Citation: Shamsutdinova GR, Viktorov SV. Solution of the inverse problem for cylindrical inclusion fragment form definition. CEUR Workshop Proceedings, 2016; 1638: 658-663. DOI: 10.18287/1613-0073-2016-1638-658-663

\section{Introduction}

Nowadays the problems of searching and exploration of mineral fields are urgent tasks of geophysics. From the practical point of view, the study of these problems is extremely important, because electrical methods of exploration are environmentally friendly for subsoil and enable people to investigate mineral deposits, estimate their size and shape most effectively. The theoretical problems, arising from the study of physical and mathematical basis of electric exploration, are searching for the form of the inclusion, and belong to the class of inverse problems of geophysics.

In this paper we solve the inverse problem of searching a deformation site of an extended cylindrical inclusion in a homogeneous medium. The solution of the problem is found in the parameters description of surface boundaries of the cylinder.

\section{Formulation of the problem}

Suppose, that in a homogeneous isotropic half-space $\Omega_{0}$ with a specific electric conductivity $\sigma_{0}$ in the plane xOy, at a distance $z=z_{0}$, parallel to the axis Ox the inclu- 
sion $\Omega_{1}$ of the extended cylindrical shape with a specific electrical conductivity $\sigma_{1}$ is located. The cylinder has a strain on the segment $x \in[a, b]$ (shift along the axis Oy) (Fig. 1). The problem can be solved if to find $a$ and $b$ parameters, which define the segment of curvature of the extended body. Such deformation may occur as a result of the shift of soil layers at landslides, earthquakes and other destructive natural phenomena.

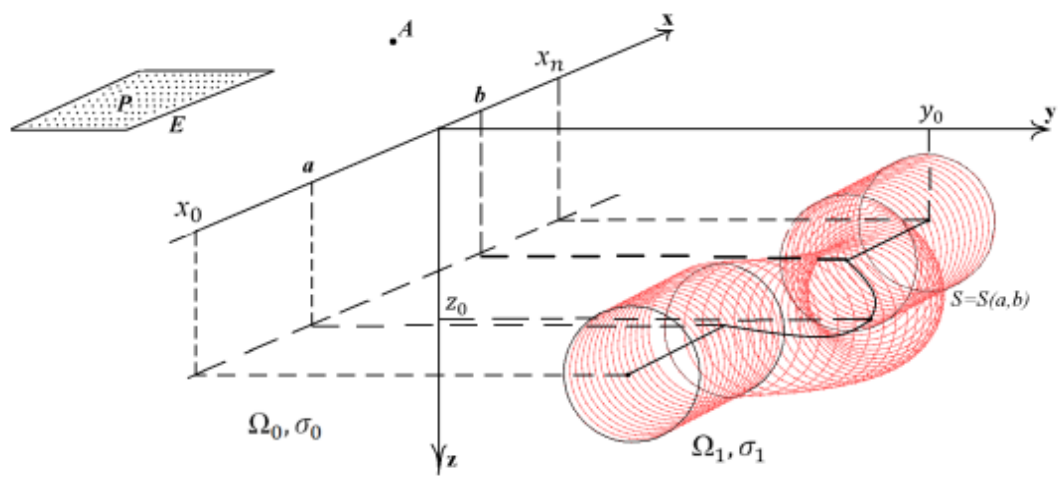

Fig. 1. The cylindrical inclusion in a homogeneous isotropic half-space

The solution of the inverse problem in searching the $[a, b]$ parameters of the inclusion $\Omega_{1}$ of the extended cylindrical shape with a specific electrical conductivity $\sigma_{1}$ can be found in search for the extremals of the A. N. Tikhonov's functional as the following $\mathrm{p} 1,3]$ :

$$
F^{\alpha}(S)=F_{1}(S)+\alpha \cdot F_{2}(S)=\left\|u(A, P, S)-u^{e}(A, P)\right\|_{L_{2}}^{2}+\alpha \cdot\left\|S-S^{e}\right\|_{W_{2}^{1}}^{2},
$$

where $S=S(a, b)$ is the function of parametric description of a deformable surface of the cylinder, $u^{e}(A, P)$ - changes in experimental geophysical data, obtained at the area of "day" surface, representing the value of the potential field of a point source of intensity of direct current, excited at the source and destination point, $u(A, P)$ - the model solution of the direct problem of the point source field is considered as the following boundary value problem of elliptic type [5,7]:

$$
\begin{aligned}
& \Delta u_{0}(A, P, S)=-\frac{I}{\sigma_{0}} \delta(P-A), P \in \Omega_{0} ; \\
& \Delta u_{1}(P, S)=0, P \notin \Omega_{0} ; \\
& \left.\frac{\partial u_{0}(P, S)}{\partial z}\right|_{z=0}=0 ; \\
& \left.u_{0}(P, S)\right|_{S}=\left.u_{1}(P, S)\right|_{S} ;
\end{aligned}
$$


$\left.\sigma_{0} \frac{\partial u_{0}(P, S)}{\partial n}\right|_{S}=\left.\sigma_{1} \frac{\partial u_{1}(P, S)}{\partial n}\right|_{S}$

$u_{0}(P, S) \rightarrow 0, P \rightarrow \infty$,

where $\Delta$ - Laplace's operator, $\delta$ - Dirac's function, condition (4) defines isolation of the Earth's surface respectively, (5) and (6) - conditions of continuity of the potential and current density respectively, (7) - regularity condition of the solution at infinity.

For the decision of tasks (2) - (7) we use the method of integral representations, based on the integral transformation with the construction of the Green's function for accommodating space $[9,10]$.

Let's choose, according to the method, a homogeneous half-space as a co-holding cylinder medium, and construct a mathematical model for it - subtask for point-source function (Green's function) $G(P, Q)$ :

$\Delta G_{Q}(Q, P)=-\delta(P-Q) ;$

$\left.\frac{\partial G_{0}}{\partial z}\right|_{z=0}=0$

$G(P) \rightarrow 0$ при $P \rightarrow \infty$.

In the case of a homogeneous half-space Green's function has the following form:

$$
\begin{aligned}
& G(P, A)=\frac{1}{4 \cdot \pi \cdot \sqrt{\left(x_{P}-x_{A}\right)^{2}+\left(y_{P}-y_{A}\right)^{2}+\left(z_{P}-z_{A}\right)^{2}}}+ \\
& +\frac{1}{4 \cdot \pi \cdot \sqrt{\left(x_{P}-x_{A}\right)^{2}+\left(y_{P}-y_{A}\right)^{2}+\left(z_{P}-z_{A^{*}}\right)^{2}}}
\end{aligned}
$$

According to the method we write the integral representation of the solution of the direct problem and the integral equation for the unknown boundary values of the potential at the boundary of the cylindrical inclusion. In order to it, let's use this formula:

$$
\begin{aligned}
& \int_{\Omega}[\Delta u(Q) \cdot G(P, Q)-\Delta G(P, Q) \cdot u(Q)] d \Omega_{Q}= \\
& =\int_{\partial \Omega}\left[\frac{\partial u(Q)}{\partial n} \cdot G(P, Q)-\frac{\partial G(P, Q)}{\partial n} \cdot u(Q)\right] d\left\{\partial \Omega_{Q}\right\} .
\end{aligned}
$$

Using it to areas $\Omega_{0}$ and $\Omega_{1}$, we get the formula (11) of the integral representation of the solution and the Fredholm integral equation of the 2nd kind (12) for the unknown boundary values of the potential $u(Q)[8]$.

$$
u(P)=\frac{\left(\sigma_{0}-\sigma_{1}\right)}{\sigma_{0}} \int_{S} \frac{\partial G(P, Q)}{\partial n} \cdot u(Q) d\{S\}+\frac{I \cdot G(P, A)}{\sigma_{0}},
$$


$u(P)=\frac{2 \cdot\left(\sigma_{0}-\sigma_{1}\right)}{\left(\sigma_{0}+\sigma_{1}\right)} \int_{S} \frac{\partial G(P, Q)}{\partial n} \cdot u(Q) d\{S\}+\frac{2 \cdot I \cdot G(P, A)}{\left(\sigma_{0}+\sigma_{1}\right)}$.

Thus, the solution is determined by the formula (11), which is a definite integral is calculated in case of the formula (12) values $u(Q)$ on the boundary S. For the numerical implementation of solutions (11) and (12) are transformed to a discrete form.

$u(P)-\lambda \int_{S} K(P, Q) \cdot u(P, Q) d\{S\}=F(P)$,

$\lambda=2 \cdot \frac{\sigma_{0}-\sigma_{1}}{\sigma_{0}+\sigma_{1}}, F(P)=2 \cdot \frac{I \cdot G(P, A)}{\sigma_{0}+\sigma_{1}}, K(P, Q)=\frac{\partial G(P, Q)}{\partial n}$.

Take the formula (13) in discrete form where the points $\mathrm{P}$ and $\mathrm{Q}$ are located in a discrete area $S_{\Delta}$.

$S \rightarrow S_{\Delta}, P \rightarrow P_{i}, Q \rightarrow Q_{j}, i, j \in[0, N]$,

$u\left(P_{i}\right)-\lambda \cdot \sum_{j=0}^{N}\left[\int_{\Delta_{j}} K\left(P_{i}, Q_{j}\right) d\left\{\Delta_{j}\right\} \cdot u\left(Q_{j}\right)\right]=F\left(P_{i}\right), i \in \overline{0, N}$

$u_{i}-\lambda \cdot \sum_{j=0}^{N} \int_{\Delta_{j}} \overline{K_{i, j}} \cdot u_{j} d\left\{\Delta_{j}\right\}=F_{i}$

where $\overline{K_{i, j}}=\int_{\Delta} K_{i, j} d\{\Delta\}, i=\overline{0, N}, N-$ the overall number of points.

The method, offered in our work, is a universal method of reducing the geometric complexity of the test environment. Besides, this method can be used to phase the complication of model geometry [2].

To solve the direct problem we used the procedure for the construction of the cylindrical inclusion [6] corresponding the formulas of parametric descriptions of the cylinder:

$x \in\left[x_{0}, x_{n}\right], \varphi \in[0,2 \pi),[a, b] \subseteq\left[x_{0}, x_{n}\right]:$

$\left\{\begin{array}{l}X(\varphi, x)=x_{0}+x, \\ Y(\varphi, x)=y_{0}+\left\{\begin{array}{l}R \cdot \sin (\varphi), \quad x \notin[a, b], \\ R \cdot \sin (\varphi)+L_{2}(x), \quad x \in[a, b]\end{array}\right. \\ Z(\varphi, x)=z_{0}+R \cdot \cos (\varphi) .\end{array}\right.$

where $i=\overline{0, N}, L_{2}(x)$ - Lagrange Interpolation polynomial function for calculating the deformation of the extended cylindrical inclusion in the segment, which is calculated according to the formula:

$c=0.5 \cdot(a+b), d=0.1 \cdot(b-a)$ :

$L_{2}(x)=\frac{(x-c) \cdot(x-b)}{(a-c) \cdot(a-b)} \cdot y_{0}+\frac{(x-a) \cdot(x-b)}{(c-a) \cdot(c-b)} \cdot\left(y_{0}+d\right)+\frac{(x-a) \cdot(x-c)}{(b-a) \cdot(b-c)} \cdot y_{0}$ 




Fig. 2. The guide cylinder with deformation

To solve the inverse problem we use the algorithm of searching for minimum functional configurations of a variable type, directed to the search for global minimum strongly ravine functions [4].

When searching for the local inclusion a cut $[a, b]$ is considered as varying parameters. In this case the algorithm of minimization method is defined as the following.

There is an initial approximation $x^{(k)}=\left(a^{(k)}, b^{(k)}\right), k=0$. Let's find $a$ and $b$ by the following way:

$a=\left\{\begin{array}{l}a^{+}, \text {если } F\left(a^{+}, \tilde{b}\right)<F(\tilde{a}, \tilde{b}), \text { где } a^{+}=\min \left(a_{\max }, a+h\right), \\ a^{-}, \text {если } F\left(a^{-}, \tilde{b}\right)<F(\tilde{a}, \tilde{b}), \text { где } a^{-}=\max \left(a_{\min }, a-h\right)\end{array}\right.$

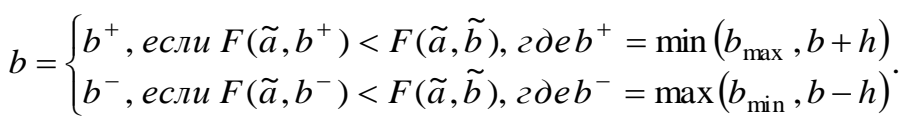

If $F(a, b)<F(\tilde{a}, \tilde{b})$, the approach taken for the value obtained by the rule $a^{(k+1)}=\tilde{a}+l(\tilde{a}-a), b^{(k+1)}=\tilde{b}+l(\tilde{b}-b)$, where $1-$ coefficient of step convergence magnification, otherwise step decreases: $h(h=h / 2)$. This process continues until the condition does not satisfy the algorithm closure: if the convergence step $h \leq \varepsilon$, then the search stops and $a^{(k+1)}, b^{(k+1)}$ is considered as a solution.

\section{Conclusion}

Software tool was used for searching the diverse problem solution and carrying out a computational experiment.

\section{References}

1. Chmielewska VC, Kostitsyn VI. Fundamentals of geophysical methods: a textbook for high schools. Perm. Univ. Perm, 2010; 400 p.

2. Dmitriev VI. Inverse Problems of Geophysics. M.: MAKS Press, 2012; 340 p.

3. Kabanikhin SI. Inverse and ill-posed problems: the textbook for students of higher educational institutions. Novosibirsk: Siberian Scientific Publishing House, 2009; 457 p.

4. Panteleev AV. Optimization methods in examples and problems. M., Higher School, 2002; $544 \mathrm{p}$.

5. Shamsutdinova GR, Viktorov SV. Direct problem of point source field in a homogeneous medium with a cylindrical inclusion. Proceedings of the IV All-Russian scientific-practical 
conference dedicated to the 75th anniversary of the Faculty of Physics and Mathematics, Part 2 "Mathematical may modeling of the processor and the system". Sterlitamak, 2015: 315-320.

6. Strelchenko VV. Geophysical exploration wells: a textbook for high schools: a textbook for high schools. M.: Nedra, 2008; 551 p.

7. Viktorov SV. Mathematical modeling geoelek-electric fields in axisymmetric environments with spline-approximation of boundaries. Diss. PhD, Sterlitamak, 2005; 106 p.

8. Wierzbicki VM. Fundamentals of numerical methods. M.: Higher School, 2009; 3rd ed.; $840 \mathrm{p}$.

9. Yagola AG, Van Yanfey, Stepanova IE, Titarenko VN. Inverse problems and recommended solutions. Applications to Geophysics, 2nd ed. M.: Binom. Knowledge Laboratory, 2014; $216 \mathrm{p}$.

10. Yanovska TB, Porohova LN. Inverse Problems of Geophysics: a tutorial. St. Petersburg. state. Univ. 2nd ed . St. Petersburg: Publishing House, St. Petersburg. Univ., 2004; 214 p. 